\title{
THE STIELTJES MOMENT PROBLEM FOR FUNCTIONS OF BOUNDED VARIATION
}

\author{
R. P. BOAS, JR.*
}

1. Introduction. We shall establish the following theorem, which at first sight appears quite unexpected:

THEOREM 1. Any sequence $\left\{\mu_{n}\right\}$ of real numbers can be represented in the form

$$
\begin{aligned}
& \mu_{n}=\int_{0}^{\infty} t^{n} d \alpha(t), \quad n=0,1,2, \cdots, \\
& \int_{0}^{\infty}|d \alpha(t)|<\infty .
\end{aligned}
$$

The problem of determining necessary and sufficient conditions for a sequence of numbers $\left\{\mu_{n}\right\}$ to have the form

$$
\mu_{n}=\int_{0}^{\infty} t^{n} d \alpha(t), \quad \alpha(t) \text { non-decreasing, } n=0,1,2, \cdots,
$$

was set and solved by T. J. Stieltjes. It would be natural to attempt to generalize the problem by requiring merely that $\alpha(t)$ should be a function of bounded variation on $(0, \infty)$; but the generalized problem has, as Theorem 1 shows, a trivial solution.

To establish Theorem 1, we shall exhibit an arbitrary real sequence $\left\{\mu_{n}\right\}$ as the difference of two sequences $\left\{\lambda_{n}\right\}$ and $\left\{\nu_{n}\right\}$, each of the form (1.2). $\dagger$ The construction will also lead to the result that any sequence $\left\{\mu^{n}\right\}$ of positive numbers of sufficiently rapid growth has the form (1.2); it is sufficient, for example, that

$$
\mu_{0} \geqq 1, \quad \mu_{n} \geqq\left(n \mu_{n-1}\right)^{n},
$$$$
n \geqq 1 \text {. }
$$

A specimen sequence satisfying (1.3) is $\mu_{0}=1, \mu_{n}=n^{n n},(n=1,2, \cdots)$. As an application $\ddagger$ of Theorem 1 , it will be shown that

* National Research Fellow.

† Added in proof: Other proofs of Theorem 1 have been given by G. Pólya (Sur l'indétermination d'un problème voisin du problème des moments, Comptes Rendus de l'Académie des Sciences, Paris, vol. 207 (1938), pp. 708-711). Pólya points out that a theorem of which Theorem 1 is an immediate consequence was proved by $\mathrm{E}$. Borel in 1894.

$\ddagger$ For another application of Theorem 1, see J. Shohat, Sur les polynomes orthogonaux généralisés, Comptes Rendus de l'Académie des Sciences, Paris, vol. 207 (1938), pp 556-558. 
with

$$
f(x)=\int_{0}^{\infty} x(t) d \alpha(t),
$$

$$
\int_{0}^{\infty} t^{n}|d \alpha(t)|<\infty, \quad n=1,2, \cdots
$$

is not the general linear functional on any very interesting space of functions $x=x(t)$, containing an infinite number of the functions $t^{n}$, $(n=1,2, \cdots)$ (see $\$ 4$ for a precise statement). Other negative results of this character have been obtained by J. W. Tukey and the author; ${ }^{*}$ the reader is referred to their paper for a discussion of the significance of such results.

2. Proof of Theorem 1. We use the notation

$$
\left[\mu_{0} \mu_{2} \cdots \mu_{2 n}\right]=\left|\begin{array}{cccc}
\mu_{0} & \mu_{1} & \cdots & \mu_{n} \\
\mu_{1} & \mu_{2} & \cdots & \mu_{n+1} \\
\cdot & \cdot & \cdots & \cdot \\
\mu_{n} & \mu_{n+1} & \cdots & \mu_{2 n}
\end{array}\right|, \quad n=0,1,2, \cdots .
$$

Then a necessary and sufficient condition for $\left\{\mu_{n}\right\}$ to have the form 1.2 is $\dagger$

(2.1) $\left[\mu_{0} \mu_{2} \cdots \mu_{2 n}\right] \geqq 0, \quad\left[\mu_{1} \mu_{3} \cdots \mu_{2 n+1}\right] \geqq 0, \quad n=0,1,2, \cdots$.

We choose positive numbers $\lambda_{0}, \lambda_{1}, \nu_{0}, \nu_{1}$, so that $\lambda_{0}-\nu_{0}=\mu_{0}$, $\lambda_{1}-\nu_{1}=\mu_{1}$. We now proceed to define the sequences $\left\{\lambda_{n}\right\},\left\{\nu_{n}\right\}$ by induction. Suppose that

$$
\lambda_{k}-\nu_{k}=\mu_{k}
$$

for $k=0,1,2, \cdots, 2 n-1$, and that the determinants

$$
\begin{array}{ll}
{\left[\lambda_{0} \lambda_{2} \cdots \lambda_{2 k}\right],} & {\left[\nu_{0} \nu_{2} \cdots \nu_{2 k}\right],} \\
{\left[\lambda_{1} \lambda_{3} \cdots \lambda_{2 k+1}\right],} & {\left[\nu_{1} \nu_{3} \cdots \nu_{2 k+1}\right],}
\end{array}
$$

are positive for $k=0,1,2, \cdots, n-1$. We have (with undetermined $\lambda_{2 n}$ )

$$
\left[\lambda_{0} \lambda_{2} \cdots \lambda_{2 n}\right]=\lambda_{2 n}\left[\lambda_{0} \lambda_{2} \cdots \lambda_{2 n-2}\right]+P,
$$

where $P$ is a polynomial in $\lambda_{0}, \lambda_{1}, \cdots, \lambda_{2 n-1}$; and there is a corre-

* R. P. Boas, Jr., and J. W. Tukey, A note on linear functionals, this Bulletin, vol. 44 (1938), pp. 523-528.

$\dagger$ See, for example, O. Perron, Die Lehre von den Kettenbrïchen, 1929, p. 410; cf. also M. Riesz, Sur le problème des moments, troisième note, Arkiv för Matematik, Astronomi och Fysik, vol. 17 (1922-1923), no. 16. 
sponding relation for $\left[\nu_{0} \nu_{2} \cdots \nu_{2 n}\right]$. Since $\left[\lambda_{0} \lambda_{2} \cdots \lambda_{2 n-2}\right]>0$, and $\left[\nu_{0} \nu_{2} \cdots \nu_{2 n-2}\right]>0$, we can choose $\lambda_{2 n}$ and $\nu_{2 n}$ so that $\lambda_{2 n}-\nu_{2 n}=\mu_{2 n}$, and so large that $\left[\lambda_{0} \lambda_{2} \cdots \lambda_{2 n}\right]>0,\left[\nu_{0} \nu_{2} \cdots \nu_{2 n}\right]>0$. Similarly we can then choose $\lambda_{2 n+1}$ and $\nu_{2 n+1}$ so that $\lambda_{2 n+1}-\nu_{2 n+1}=\mu_{2 n+1}$, $\left[\lambda_{1} \lambda_{3} \cdots \lambda_{2 n+1}\right]>0,\left[\nu_{1} \nu_{3} \cdots \nu_{2 n+1}\right]>0$. This completes the induction: we can find sequences $\left\{\lambda_{n}\right\},\left\{\nu_{n}\right\}$ such that for $k=0,1,2, \cdots$, (2.2) is satisfied, and all the determinants (2.3) are positive. Then $\left\{\lambda_{n}\right\}$ and $\left\{\nu_{n}\right\}$ satisfy (2.1), and consequently have the form (1.2), so that $\left\{\mu_{n}\right\}$ has the form (1.1).

3. Rapidly increasing sequences. We now prove the following theorem:

TheOREM 2. If

$$
\mu_{0} \geqq 1, \quad \mu_{n} \geqq\left(n \mu_{n-1}\right)^{n},
$$$$
n=1,2, \cdots,
$$

then $\left\{\mu_{n}\right\}$ has the form (1.2).

For the proof, we modify the construction of the sequence $\left\{\lambda_{n}\right\}$ of $\S 2$. We have, for $n=1,2, \cdots$,

$$
\left[\mu_{0} \mu_{2} \cdots \mu_{2 n}\right]=\mu_{2 n}\left[\mu_{0} \mu_{2} \cdots \mu_{2 n-2}\right]+\sum_{k=n}^{2 n-1} \pm \mu_{k} D_{k},
$$

where the $D_{k}$ are $n$-rowed minors of $\left[\mu_{0} \mu_{2} \cdots \mu_{2 n}\right]$ and do not involve $\mu_{2 n}$. Similarly, for $n=1,2, \cdots$,

$$
\left[\mu_{1} \mu_{3} \cdots \mu_{2 n+1}\right]=\mu_{2 n+1}\left[\mu_{1} \mu_{3} \cdots \mu_{2 n-1}\right]+\sum_{k=n+1}^{2 n} \pm \mu_{k} D_{k}^{\prime}
$$

where the $D_{k}^{\prime}$ are $n$-rowed minors of $\left[\mu_{1} \mu_{3} \cdots \mu_{2 n+1}\right]$, not involving $\mu_{2 n+1}$.

Suppose that for $k \leqq n-1,(n=1,2, \cdots)$,

$$
\left[\mu_{0} \mu_{2} \cdots \mu_{2 k}\right] \geqq 1, \quad\left[\mu_{1} \mu_{3} \cdots \mu_{2 k+1}\right] \geqq 1 .
$$

Assuming (3.1), we shall show that (3.4) is satisfied also for $k=n$.

Clearly, $\mu_{m} \geqq 1$ for $m=1,2, \cdots$. Hence we have

$$
\mu_{m} \geqq\left(m \mu_{m-1}\right)^{m}>2(m / 2)^{(m+4) / 4} \mu_{m-1}^{(m+2) / 2}, m=2,3, \cdots .
$$

Therefore

$$
\mu_{2 n}>1+n^{(n+2) / 2} \mu_{2 n-1}^{n+1}, \quad \mu_{2 n+1}>1+n^{(n+2) / 2} \mu_{2 n}^{n+1} .
$$

Now, (3.1) implies in particular that $\mu_{m+1} \geqq \mu_{m},(m \geqq 1)$; hence the elements of the determinants $D_{k}$ do not exceed $\mu_{2 n-1}$, and the ele- 
ments of the $D_{k}^{\prime}$ do not exceed $\mu_{2 n}$. Then by Hadamard's theorem,*

$$
\begin{aligned}
\left|D_{k}\right| & \leqq \mu_{2 n-1}^{n} n^{n / 2}, & k & =n, n+1, \cdots, 2 n-1, \\
\left|D_{k}^{\prime}\right| & \leqq \mu_{2 n}^{n} n^{n / 2}, & k & =n+1, n+2, \cdots, 2 n .
\end{aligned}
$$

Therefore, using (3.2), (3.3), (3.4), (3.5), we obtain

$$
\begin{aligned}
{\left[\mu_{0} \mu_{2} \cdots \mu_{2 n}\right] } & \geqq \mu_{2 n}-n^{1+n / 2} \mu_{2 n-1}^{n+1}>1, \\
{\left[\mu_{1} \mu_{3} \cdots \mu_{2 n+1}\right] } & \geqq \mu_{2 n+1}-n^{1+n / 2} \mu_{2 n}^{n+1}>1 .
\end{aligned}
$$

Thus (3.4) holds for $k=n$ if it holds for $k<n$; but it holds for $k=0$ by assumption, and consequently holds for all $k$; therefore $\left\{\mu_{n}\right\}$ has the form (1.2).

The moment problem (1.2) is said to be determined or undetermined according as the function $\alpha(t)$ is or is not unique (after being normalized by the conditions $\alpha(0)=0, \alpha(t)=[\alpha(t+)+\alpha(t-)] / 2$ for $t>0)$. A consequence of Theorem 2 is that the moment problem (1.2) is not only solvable for any sequence $\left\{\mu_{n}\right\}$ of sufficiently rapid growth, but is even undetermined. In fact, if $\left\{\mu_{n}\right\}$ satisfies (3.1) and if in addition $\mu_{2} \geqq\left(2 \mu_{1}+2\right)^{2}$, we define a sequence $\left\{\nu_{n}\right\}$ by setting $\nu_{1}=\mu_{1}+1, \nu_{n}=\mu_{n}$ for $n \neq 1$. Then $\left\{\nu_{n}\right\}$ satisfies (3.1); consequently for $n=0,1,2, \cdots$,

$$
\nu_{2 n}=\int_{0}^{\infty} t^{2 n} d \beta(t)=\int_{0}^{\infty} u^{n} d \beta\left(u^{1 / 2}\right)=\int_{0}^{\infty} u^{n} d \gamma(u),
$$

say; while

$$
\nu_{2 n}=\mu_{2 n}=\int_{0}^{\infty} t^{2 n} d \alpha(t)=\int_{0}^{\infty} u^{n} d \delta(u),
$$

where $\gamma(u)$ and $\delta(u)$ are normalized and non-decreasing. But $\gamma(u)$ and $\delta(u)$ are distinct, since

$$
\nu_{1}=\int_{0}^{\infty} u^{1 / 2} d \gamma(u)=1+\int_{0}^{\infty} u^{1 / 2} d \delta(u)=1+\mu_{1} .
$$

Hence the moment problem for the sequence $\left\{\mu_{2 n}\right\}$ is undetermined.

4. Linear functionals. We use the terminology of S. Banach's book. $\dagger$ Let $R$ be a topological vector space of elements $x$, let $P$ be a

* G. H. Hardy, J. E. Littlewood, and G. Pólya, Inequalities, 1934, p. 34.

$\dagger$ Thêorie des Opérations Linéaires, 1932. 
space of elements $p$, and let $f_{p}(x)$ be a functional with domain $R$, defined for each $p$ in $P$. We say that a general linear functional in $R$ is $f_{p}(x)$, if the following conditions are satisfied:

(i) $f_{p}(x)$ is a linear functional for every $p \varepsilon P$.

(ii) Every linear functional $g(x)$ with domain $R$ is identically equal to some $f_{p}(x)$.

In the application to be made here, the elements of $P$ are the functions $p=p(t)$, of bounded variation on $(0, \infty)$, such that

$$
\int_{0}^{\infty} t^{n}|d p(t)|<\infty, \quad n=1,2, \cdots ;
$$

the elements of $R$ are measurable functions $x=x(t)$, defined on $(0, \infty)$; and

$$
f_{p}(x)=\int_{0}^{\infty} x(t) d p(t)
$$

where the integral is a Lebesgue-Stieltjes integral. We have the following result:

THEOREM 3. Let $R$ be a topological vector space with the following property:*

(Q): If $x \in R$ and $a_{n} \rightarrow 0$, then $a_{n} x \rightarrow \Theta . \dagger$

Then if $R$ contains an infinite number of functions $t^{n},(n=0,1$, $2, \cdots)$, there is some $p \varepsilon P$ for which (4.1) is not a linear functional on $R$.

In particular, we see that, under the hypotheses of Theorem 3, (4.1) is not a general linear functional on $R$.

Suppose that (4.1) is, for every $p \varepsilon P$, a linear functional on a space $R$ with the specified properties. Let $S$ be the subspace composed of all finite linear combinations of the elements $t^{n}$ which are in $R$ (with the topology of $R$ ). If $f$ is an arbitrary distributive (that is, additive and homogeneous) functional with domain $S$, we define a sequence $\left\{\mu_{n}\right\}$ by setting $\mu_{n}=f\left(t^{n}\right)$ when $t^{n} \varepsilon R$, and $\mu_{n}=0$ otherwise. By Theorem 1 , there is a $p \varepsilon P$ such that

$$
\mu_{n}=\int_{0}^{\infty} t^{n} d p(t), \quad n=0,1,2, \cdots .
$$

Since $f$ is distributive, we then have

* In particular, a space of type $F$ has this property.

$\dagger \Theta$ denotes the zero element of $R$. 


$$
f(x)=\int_{0}^{\infty} x(t) d p(t)
$$

$x \varepsilon S$.

Now (4.1) is a linear functional on $R$, and consequently a linear functional on $S$. Hence (4.2) states that every distributive functional on $S$ is linear; but this is impossible unless $S$ is finite-dimensional,* which it is not. This contradiction establishes the theorem.

Norton, Massachusetts

\section{ON FUNDAMENTAL SYSTEMS OF SYMMETRIC FUNCTIONS $\uparrow$}

\section{H. T. ENGSTROM}

A set $S$ of $n$ polynomials over a field $K$, symmetric in $n$ variables, $x_{1}, x_{2}, \cdots, x_{n}$, is said to form a fundamental system if any rational function over $K$, symmetric in these variables, can be expressed rationally in terms of the polynomials of $S$. In this paper we show that any $n$ algebraically independent symmetric polynomials over a field $K$ of characteristic zero form a fundamental system if the product of their degrees is less than $2 n$ !.

The result follows from a theorem due to Perron : $\ddagger$

Theorem 1. Between $n+1$ polynomials (not constant), $f_{1}, f_{2}, \cdots, f_{n+1}$, in $n$ variables, of degrees $m_{1}, m_{2}, \cdots, m_{n+1}$, respectively, there is always an identity of the form

$$
\sum C_{\nu_{1} \nu_{2}} \cdots \nu_{n+1} f_{1}^{\nu_{1}} f_{2}^{\nu_{2}} \cdots f_{n+1}^{\nu_{n+1}} \equiv 0
$$

where in each term,

$$
\sum_{i=1}^{n+1} m_{i} \nu_{i} \leqq \prod_{i=1}^{n+1} m_{i}
$$

* Let every distributive functional on $S$ be linear, where $S$ is a topological vector space with the property $(Q)$. If $S$ is infinite dimensional, let $\left\{x_{n}\right\},(n=1,2, \cdots)$, be an infinite set of linearly independent elements. Since $\lim _{k \rightarrow \infty} k^{-1} x_{n}=\Theta$, we can choose $y_{n} \varepsilon S,(n=1,2, \cdots)$, linearly independent, with $y_{n} \rightarrow \Theta$. We set $f\left(y_{n}\right)=1, f(x)=0$ when $x$ is not a finite linear combination of the $y_{n}, f(a x+b y)=a f(x)+b f(y)$ for any $x \in S, y \varepsilon S$; then $f$ is a distributive functional on $S$, and hence is linear on $S$. Since $y_{n} \rightarrow \Theta, f\left(y_{n}\right) \rightarrow 0$ as $n \rightarrow \infty$; but this contradicts $f\left(y_{n}\right)=1$. Consequently $S$ is finite dimensional.

$\dagger$ Presented to the Society, February 25, 1939, under the title $A$ note on fundamental systems of symmetric functions.

$\ddagger$ O. Perron, Bemerkung zur Algebra, Sitzungsberichte der Bayerischen Akademie, mathematisch-naturwissenschaftliche Abteilung, 1924, pp. 87-101. 